\title{
Exploration of the relationship between intestinal flora changes and gut acute graft-versus-host disease after hematopoietic stem cell transplantation
}

\author{
Aiyun Song ${ }^{1 \#}$, Nan Shen ${ }^{2 \#}$, Chi Gan ${ }^{2}$, Changying Luo ${ }^{1}$, Chengjuan Luo ${ }^{1}$, Jianmin Wang ${ }^{1}$, Qing Cao ${ }^{2}$, \\ Jing Chen ${ }^{1}$
}

${ }^{1}$ Department of Hematology and Oncology, Shanghai Children's Medical Center, Shanghai Jiao Tong University School of Medicine, Shanghai, China; ${ }^{2}$ Department of Infectious Disease, Shanghai Children's Medical Center, Shanghai Jiao Tong University School of Medicine, Shanghai, China Contributions: (I) Conception and design: A Song; (II) Administrative support: J Chen, Q Cao; (III) Provision of study materials or patients: A Song, C Gan, C Luo, C Luo, Q Cao, J Wang; (IV) Collection and assembly of data: A Song, C Gan; (V) Data analysis and interpretation: A Song, N Shen; (VI) Manuscript writing: All authors; (VII) Final approval of manuscript: All authors.

"These authors contributed equally to this work.

Correspondence to: Jing Chen. Department of Hematology and Oncology, Shanghai Children's Medical Center, Shanghai Jiao Tong University, School of Medicine, No. 1678, Dongfang Road, Shanghai 200127, China. Email: chenjing@scmc.com.cn; Qing Cao. Department of Infectious Disease, Shanghai Children's Medical Center, Shanghai Jiao Tong University, School of Medicine, No. 1678, Dongfang Road, Shanghai 200127, China. Email: caoqing@scmc.com.cn.

Background: Acute graft-versus-host disease (aGVHD) is a life-threatening factor for post-hematopoietic stem cell transplantation (HSCT) patients. To investigate the relationship between intestinal flora changes and gut aGVHD after HSCT, we performed this cross-sectional study.

Methods: We selected children from our medical center from July 2016 to January 2017. Fifty-six samples from 42 patients and 6 samples from normal children met the study criteria and were analyzed. Fecal 16S RNA sequencing was completed before transplantation or on days 7, 28 or 100 post-transplantation. The intestinal infection and GVHD clinical data were retrospectively analyzed, and the survival risk factors were analyzed. Correlation analysis was performed with the feces bioinformatic data.

Results: The GVHD group alpha diversity was the lowest, which was significantly different than that of the non-diarrhea group ( $\mathrm{P}$ value=0.032). A richer posttransplantation relative abundance of Moraxellaceae was conducive to survival, while that of Enterococcaceae and Alphaproteobacteria was not. Similarly, a rich relative abundance of Proteobacteria, Gammaproteobacteria and Odoribacteraceae in the intestinal flora before HSCT contributed to patient death thereafter. Regarding diarrhea, the GVHD group exhibited a richer Pasteurellales and Pasteurellaceae relative abundances, which showed strong correlations with diarrhea severity. Peptostreptococcaceae, Bifidobacteriales and Bifidobacteriaceae were richer in relative abundance in the intestinal infection group and correlated with pretransplant characteristics.

Conclusions: The gut microbiota diversity was lowest when gut aGVHD occurred, which was consistent with the clinically higher mortality rate and greater treatment difficulty. Pasteurellaceae played an important role in gut aGVHD and diarrhea severity. Bifidobacteriaceae led to infectious diarrhea after HSCT. Specific bacteria were biomarkers for survival: Moraxellaceae, Enterococcaceae and Alphaproteobacteria from the intestinal flora after HSCT and Proteobacteria, Gammaproteobacteria and Odoribacteraceae before HSCT.

Keywords: Hematopoietic stem cell transplantation (HSCT); gut acute graft-versus-host disease (gut aGVHD) GVHD; posttransplant infection; diarrhea; intestinal microbiome

Submitted Jul 24, 2020. Accepted for publication Dec 04, 2020.

doi: $10.21037 / \mathrm{tp}-20-208$

View this article at: http://dx.doi.org/10.21037/tp-20-208 


\section{Introduction}

Hematopoietic stem cell transplantation (HSCT) is a potential curable treatment for various malignant blood diseases and genetic diseases and is divided into two steps: conditioning regimen therapy and stem cell infusion. In practice, a fully or partially myeloablative conditioning regimen depletes malignant or deficient hematopoietic cells as well as healthy hematopoietic cells. After the regimen, patients will immediately experience a transient but profound state of immune deficiency, which greatly increases the risk of infection. HSCT patients will also receive a therapy of immunosuppression according to the process. The decline in immune function was inevitable and influenced by many facts. HLA-mismatched donor sources will lead to a more intensive myeloablative conditioning regimen and a longer period of immunosuppression state. Meanwhile, stem cell engraftment starts, patients face many post-transplant complications like infection, engraftment syndrome, acute graft-versus-host disease (GVHD) (in the skin, liver, and gut, manifesting as fever, rash, jaundice, and diarrhea) and chronic GVHD (in the skin, liver, and lung), which can lead to engraftment failure, disease relapses and even death. Physicians work hard in the choice of donor sources, conditioning regimen and immunosuppressive regimen to avoid a fatal complication.

Diarrhea is one of the most common symptoms of the digestive system after transplantation and seriously affects the prognosis of treatment. Isolated anti-infective treatment alone cannot improve gut aGVHD, while the use of increasing levels of immunosuppressive agents often leads to fatal infections. Gut aGVHD can account for $60 \%$ of the total incidence of aGVHD (1) and is the leading cause of aGVHD-related death. Clinically, infection and gut aGVHD can occur at the same time, thus increasing the difficulty of treatment, especially before the completion of immune reconstitution and in the absence of a pathogenic diagnosis or pathological findings of the intestinal mucosa. In practice, before the pathogenic results emerge, for fear of the harm of GVHD, physicians will prioritize an increase in the dose of immunosuppressive drugs, which will aggravate the infection.

Diarrhea complications are mostly incompletely understood. However, growing evidence suggests that the microbiota plays an important role in the prediction of severe GVHD and transplant-related mortality (TRM) $(2,3)$. Currently, mechanisms of the microbiota in GVHD have been reported as the immunoregulation function itself and that of its metabolic production, resulting in a vicious circle of microbiota imbalance and gut aGVHD revealed by numerous studies in adults. Among the microbial studies in the HSCT field published so far, one study of HSCT in children has been conducted to review the prophylaxis of GVHD in 112 children with gastrointestinal decontamination (4). It is believed that the intestinal flora is related to race, age, body mass index [BMI (5)], etc. Therefore, the distribution of the intestinal flora in children is different from that in adults. Due to growth and development, children require better digestion and absorption function of the intestine than adults. Therefore, we aimed to investigate HSCT-related intestinal flora changes in children and their effects on gut aGVHD.

We present the following article in accordance with the STROBE reporting checklist (available at http://dx.doi. org/10.21037/tp-20-208).

\section{Methods}

\section{Patient selection}

We conducted a cross-sectional study from July 2016 to January 2017 at Shanghai Children's Medical Center. The disease group came from the Hematological and Oncology Department, and the healthy control group came from the outpatient department. The inclusion criteria included the following: (I) patients completed HSCT and evaluated the success of engraftment at 100 days after transplantation; (II) stool samples collected before or at 7, 28, or 100 days after stem cell infusion; (III) clinical case data and followup information had high integrity; (IV) healthy children had no allergic history or antibiotic use within half a year. Since the collection of fecal samples could not be artificially interfered, they were difficult to collect. Therefore, under the above conditions, a total of 87 HSCT samples and 6 healthy child samples met the criteria, of which 22 stool samples were not qualified for sequencing and 9 samples were rejected for antibiotic use within 2 weeks before transplantation (Figure 1).

The study was conducted in accordance with the Declaration of Helsinki (as revised in 2013). The studies involving human participants were reviewed and approved by the Human Ethics Committee of Shanghai Children's Medical Center (No. SCMCIRB-K2016053-2). Written informed consent to participate in this study was provided by the participants' legal guardian/next of kin. 


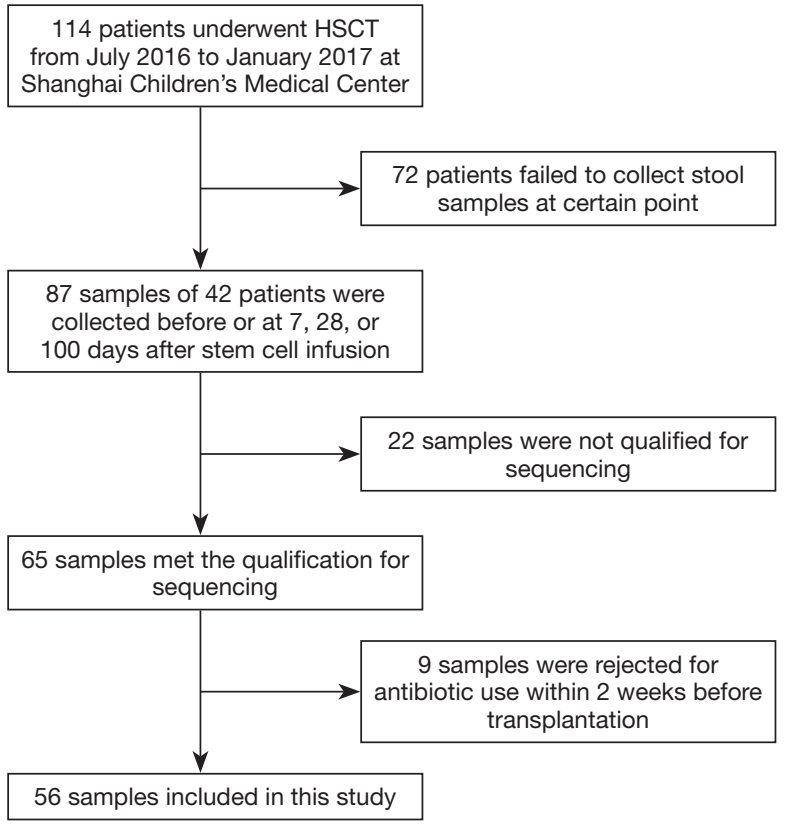

Figure 1 Flow chart of sample selection.

\section{Patient features}

Finally, a total of 56 samples from 42 patients met the criteria. The mean age was $6.3 \pm 4.08$ years (range, 9 months to 17 years); 26 were males $(61.9 \%)$; the mean followup period for surviving patients was $497 \pm 242$ days (range, 34-767 days), and the mean BMI was 16.6 \pm 2.33 (range, 11.9-22.4). Our patients varied regarding underlying diseases, which significantly differed from adult HSCT recipients, who mostly suffer from malignant diseases, such as leukemia or myelodysplastic syndrome. The underlying diseases are presented in the following pie chart (Figure 2). Patients underwent HSCT from different kinds of donor sources (Figure 3) and received conditioning regimen therapy separately (Table 1). Methotrexate and cyclosporin A were given as prophylaxis from GVHD for all patients. In terms of antiinfection, all patients were given fluconazole for prophylaxis of fungal infections; A broadspectrum prophylactic antimicrobial treatment over both gram-stain-positive and negative bacteria was given before

$1.36 \%$ Acute lymphoblastic leukemia (ALL)

13.64\% Acute nonlymphocytic leukemia (AML)

2.27\% Juvenile myelomonocytic leukemia (JMML)

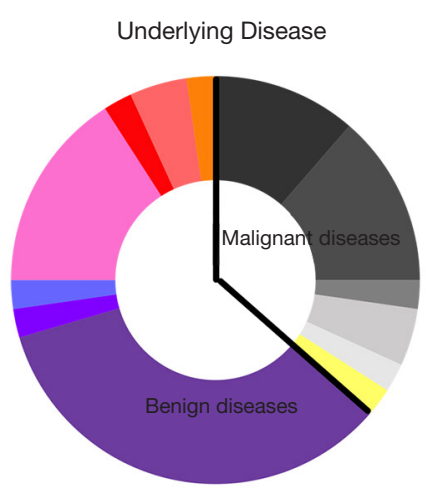

4.55\% Myelodysplastic syndrome (MDS)

$2.27 \%$ Lymphoma

$2.27 \%$ Neuroblastoma

$34.09 \%$ Aplastic anemia

$2.27 \%$ Fanconi anemia

$2.27 \%$ Thalassemia

15.91\% Mucopolysaccharidosis (MPS)

$2.27 \%$ Chronic granulomatous disease (CGD)

4.55\% X-linked high IgM syndrome (X-HIgM)

2.27\% Wiscot-Aldrich syndrome (WAS)

Figure 2 Pie chart of underlying disease percentages. 


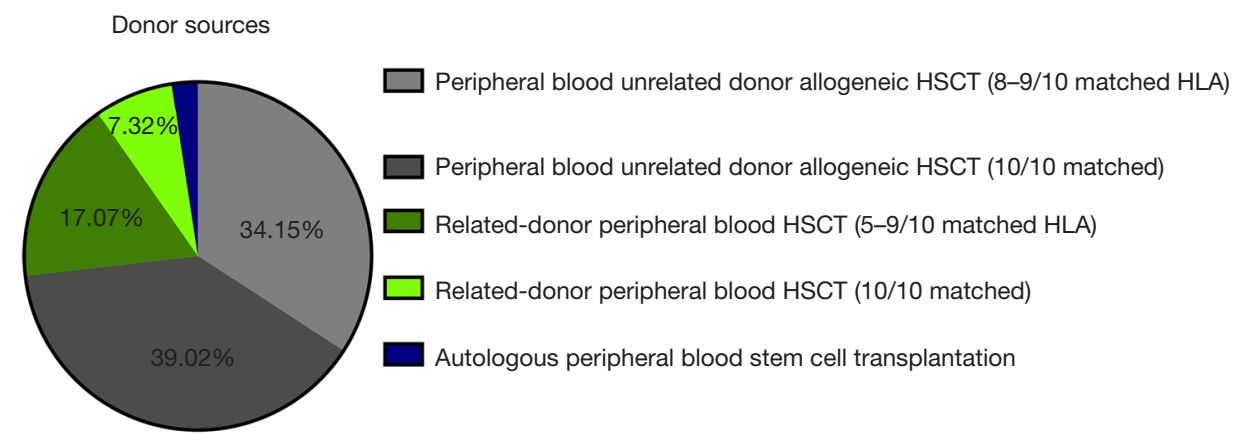

Figure 3 Pie chart of donor source percentages. HSCT, hematopoietic stem cell transplantation; HLA, human leukocyte antigen.

Table 1 Different conditioning regimen therapies

\begin{tabular}{ll}
\hline Conditioning regimen therapy & Underlying disease \\
\hline $\mathrm{BU}+\mathrm{VP1} 16+\mathrm{CTX}+\mathrm{ATG}$ & ALL (patients less than 3 years old) \\
$\mathrm{TBI}+\mathrm{VP16}+\mathrm{CTX}+\mathrm{ATG}$ & ALL (patients greater than 3 years old) \\
$\mathrm{BU}+\mathrm{CTX}+\mathrm{ATG}$ & AML, JMML, MDS, MPS, CGD, X-HIgM, WAS \\
$\mathrm{FLU}+\mathrm{CTX}+$ ATG & Aplastic anemia \\
$\mathrm{BU}+\mathrm{FLU}+\mathrm{CTX}+$ ATG & Fanconi anemia \\
$\mathrm{TBI}+\mathrm{CTX}+$ ATG & Lymphoma \\
$\mathrm{BU}$ & Neuroblastoma \\
\hline
\end{tabular}

BU, busulfan; VP16, etoposide; CTX, cyclophosphamide; ATG, anti-thymocyte globulin; FLU, fludarabine; and TBI, total body irradiation; ALL, acute lymphoblastic leukemia; AML, acute myeloid leukemia; JMML, juvenile myelomonocytic leukemia; MDS, myelodysplastic syndrome; MPS, mucopolysaccharidosis; CGD, chronic granulomatous disease; X-HIgM, X-linked high lgM syndrome; WAS, WiskottAldrich syndrome.

immune reconstitution, and antibiotic prophylaxis against Pneumocystis carinii was also generally administered.

\section{Clinical data}

All clinical data regarding intestinal infection and gut aGVHD were determined by retrospective review of the clinical charts, with individuals blinded to the microbiome results of the participants. In combination with the characteristics of clinical manifestations and the exclusion of other diarrhea causes, gut aGVHD can be diagnosed, graded according to Glucksberg grading and IBMTR criteria (6), and treated with glucocorticoids. If diarrhea progresses acutely after 3 days of glucocorticoid therapy or if the symptoms are not relieved after 7 days (7), it is defined as "glucocorticoid-resistant acute GVHD", and a second-line treatment plan should be considered. Intestinal infection is diagnosed with fever, diarrhea, and quick response to antibiotics within 48 hours. Positive pathogen results were not required because prophylactic broad-spectrum antibiotic therapy was introduced routinely when patients' absolute neutrophil count was below 500 cells $/ \mathrm{mm}^{3}$, which would undermine bacterial culture findings.

A total from 56 samples of 42 patients and 6 samples from normal children met the study criteria and were selected for analysis. Twenty-nine patient samples were collected before transplantation and 27 after transplantation. Twenty-seven posttransplantation samples included those from 25 children, 2 of which belonged to the same child at different GVHD stages ( +30 days and +60 days), and 2 other samples were from the same child under different causes of diarrhea. We grouped participants into 3 segments according to the causes of diarrhea (Figure 4). Importantly, participants in our study varied regarding underlying diseases, which was different from adult patients, who mainly suffer from malignant blood diseases. However, 
as shown in Tables 2,3, patients' characteristics, especially HSCT-related characteristics, displayed no differences between diarrhea causes.

\section{Fecal sample bioinformatics analysis}

The fecal samples of the disease group and healthy people were sampled and stored in a biofreezer at $-80{ }^{\circ} \mathrm{C}$. All sample DNA was extracted by PCR and qualified.

Taxonomic ranks were assigned to operational taxonomic unit (OTU) representative sequences. Alpha diversity analysis was applied to analyze the complexity of species (9)

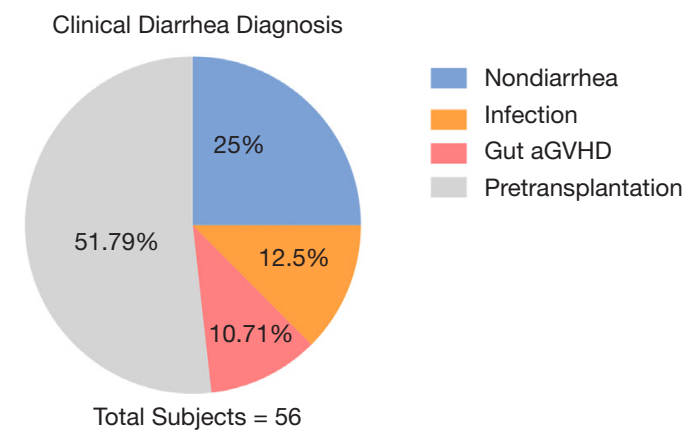

Figure 4 Pie chart of clinical diarrhea diagnosis percentages. aGVHD, acute graft-versus-host disease. diversity for a sample through several indices, including Simpson and Faith's phylogenetic diversity (PD). Beta diversity analysis was used to evaluate differences in samples in species complexity. Unweighted UniFrac distance was used to measure beta diversity. All analyses above were analyzed based on OTU and taxonomic ranks.

\section{Statistical analysis}

Descriptive statistics (mean, median, standard deviation, and discrete range) were used to show the epidemiological characteristics of the patients. For the significance test of independent samples that did not conform to the normal distribution, the chi-square test method was applied. The Kaplan-Meier (KM) method was used to calculate the overall survival (OS) rate. In the survival data, the date of HSCT was defined as time zero, and the last follow-up date was the time of censoring. All of the patient characteristics were considered potential influencing factors, and univariate analysis was performed to calculate the impact factors for diarrhea using Fisher's exact test. Correlation analysis between specific bacterial strains and clinical data was performed using Pearson correlation coefficient (satisfying normal distribution) and Spearman correlation coefficient analysis. Statistical analysis was performed using SPSS version 24.0 (IBM, New York, USA). Bioinformatic results

Table 2 Pretransplant characteristics of study participants (clinical classification of diarrhea*, post-transplantation) $\left(\mathrm{N}^{\ell}=26\right)$

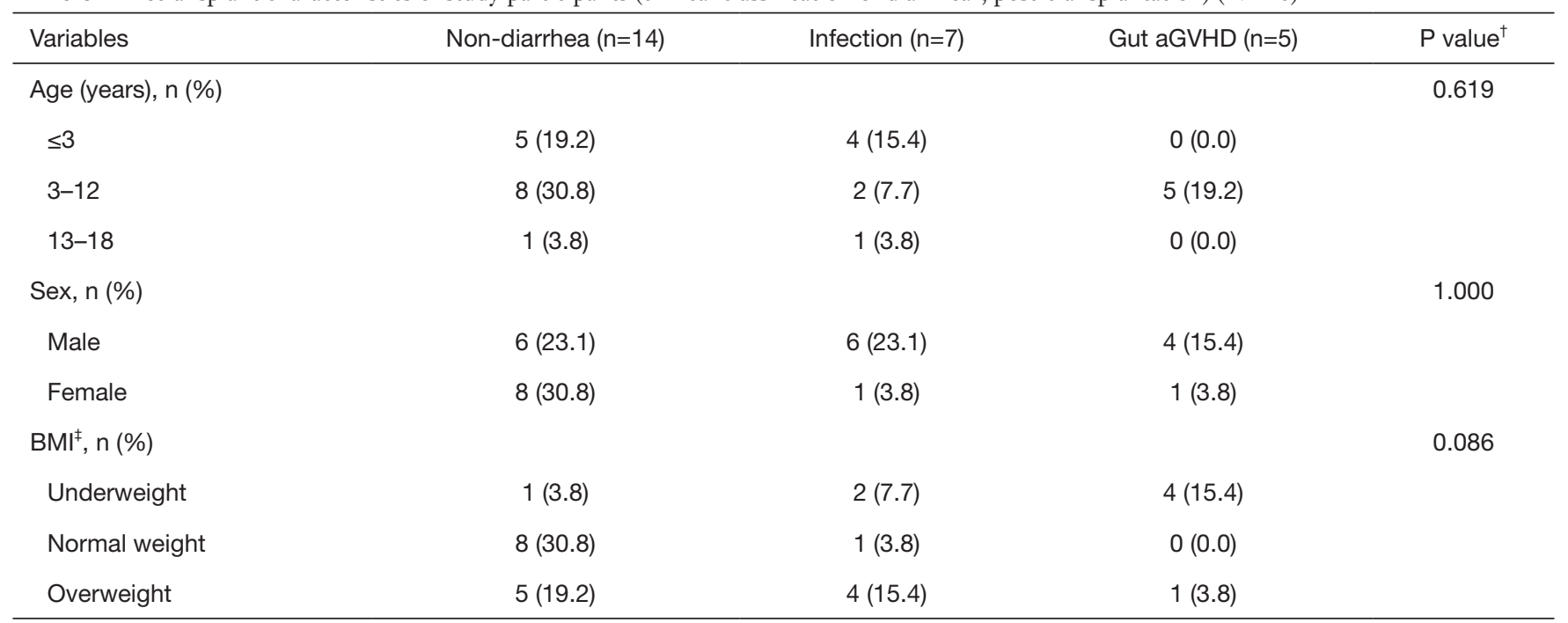

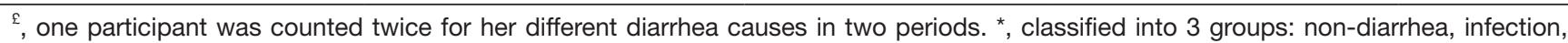
and gut aGVHD. ${ }^{\dagger}, \mathrm{P}$ values are calculated between the infection and aGVHD groups, which are 2-sided and based on Fisher's exact test. ${ }^{\ddagger}$, $\mathrm{BMI}$ is based on BMI-for-age published by the Ministry of Public Health of China in 2009 (8). N, number; aGVHD, acute graft-versus-host disease. 
Table 3 Transplant characteristics of study participants (clinical classification of diarrhea*, post-transplantation) $\left(\mathrm{N}^{\mathcal{L}}=26\right)$

\begin{tabular}{|c|c|c|c|c|}
\hline Variables & Non-diarrhea $(n=14)$ & Infection $(n=7)$ & Gut aGVHD $(n=5)$ & $P$ value ${ }^{\dagger}$ \\
\hline Myeloablative with TBI radiation & 9 & 7 & 3 & \multirow{2}{*}{0.417} \\
\hline Myeloablative without TBI radiation & 5 & 0 & 2 & \\
\hline \multicolumn{4}{|l|}{ Stem cell source } & \multirow[t]{5}{*}{1.000} \\
\hline MMRD & 2 & 2 & 2 & \\
\hline MUD & 7 & 1 & 1 & \\
\hline MMUD & 4 & 3 & 2 & \\
\hline Autologous & 0 & 1 & 0 & \\
\hline Benign diseases" & 11 & 5 & 1 & 0.242 \\
\hline \multicolumn{5}{|c|}{$\begin{array}{l}\text { £, one participant was counted twice for her different diarrhea causes in two periods. * } \text {, classified into } 3 \text { groups: non-diarrhea, infection, } \\
\text { and gut aGVHD. }{ }^{\dagger}, \mathrm{P} \text { values are calculated between infection and aGVHD groups, which are } 2 \text {-sided and based on Fisher's exact test. } \S \text {, } \\
\text { malignant diseases included leukemia, myelodysplastic syndrome, lymphoma, and neuroblastoma. ", benign diseases included aplastic } \\
\text { anemia, Fanconi anemia, thalassemia, mucopolysaccharidosis, chromosomal gonadal digenesis, X-linked hyper-lgM syndrome and } \\
\text { Wiskott-Aldrich syndrome. N, number; MSD, HLA type matching sibling donor; MMRD, HLA type miss-matching related donor; MUD, HLA } \\
\text { type matching unrelated donor; MMUD, HLA type miss-matching unrelated donor; aGVHD, acute graft-versus-host disease. }\end{array}$} \\
\hline
\end{tabular}

were analyzed using Metastats and R software (rank sum test, Fisher's exact test, chi-square test, $t$-test and variance test) for significant difference analysis between groups. The $\mathrm{P}$ value was considered statistically significant under 0.05 . We adjusted the obtained $\mathrm{P}$ value by a Benjamini-Hochberg false discovery rate correction [function 'P. adjust' in the stats package of $\mathrm{R}(\mathrm{v} 3.1 .1)]$.

\section{Results}

\section{Diarrbea symptoms became worse in the gut GVHD group}

We classified participants into 3 groups: non-diarrhea, infection, and gut aGVHD. Diarrhea symptoms were presented by diarrhea severity, volume, fever and lablaboratory examinations of C-reactive protein levels and regulatory $\mathrm{T}$ cell counts and percentages (Table 4). However, none of them was the ideal marker of diarrhea differentiation, unfortunately. Nevertheless, we observed a trend of more severe symptoms in the GVHD group by higher grade of severity and larger volume. Therefore, we hypothesize that the components and diversity of fecal microorganisms might differ between groups and interact with diarrhea symptoms by metabolic and molecular signaling pathways. Therefore, we began microbial research directly related to diarrhea, looking for a breakthrough.

\section{Taxonomic analysis of the gut microbial structure of patients}

We firstly started with a bioinformatic analysis of fecal specimen from the patients. As shown in Figure 5, all preand post-transplant specimens were analyzed in genus level and listed by number. Bacteroides dominated the contents of gut microbiome in most patients' samples while other bacterium distributed differently from each other. We therefore conducted further bioinformatics analysis to find out what influenced the symptom of diarrhea in gut aGVHD patients.

\section{Species diversity declined most in the gut aGVHD group}

We studied the microbiome in 2 ways: the difference in species composition between groups (beta diversity) and mean species diversity in groups (alpha diversity). The alpha diversity (Figure $6 A$ ) of the pre-HSCT group was significantly different from that of the post-HSCT group 
Table 4 Diarrhea symptoms and clinical data (clinical classification of diarrhea*, post-transplantation) $\left(\mathrm{N}^{\varepsilon}=27\right)$

\begin{tabular}{|c|c|c|c|c|}
\hline Variables & Non-diarrhea $(n=14)$ & Infection $(n=7)$ & Gut aGVHD $(n=6)$ & $P$ value ${ }^{\dagger}$ \\
\hline Grade 0 & $14(51.9)$ & $1(3.7)$ & $0(0.0)$ & \\
\hline Grade I & $0(0.0)$ & $3(1.1)$ & $1(3.7)$ & \\
\hline Grade II & $0(0.0)$ & $3(1.1)$ & $3(1.1)$ & \\
\hline Forehead temperature, $\mathrm{n}$ & & & & 0.155 \\
\hline$<37^{\circ} \mathrm{C}$ & 14 & 1 & 4 & \\
\hline $37-38.5^{\circ} \mathrm{C}$ & 0 & 3 & 2 & \\
\hline$>38.5^{\circ} \mathrm{C}$ & 0 & 3 & 0 & \\
\hline Elevated & 2 & 6 & 3 & \\
\hline Volume (mean values), $\mathrm{mL}$ & $31.57 \pm 12.653$ & $427.29 \pm 365.991$ & $809.5 \pm 591.009$ & 1.000 \\
\hline Regulatory T cell (mean values), \% & $3.037 \pm 3.770$ & $2.160 \pm 0.154$ & $2.945 \pm 3.002$ & 0.353 \\
\hline Regulatory $\mathrm{T}$ cell ${ }^{\#}$ (mean values), $\mu \mathrm{L}$ & $51.376 \pm 31.565$ & $51.880 \pm 50.531$ & $41.315 \pm 25.455$ & 0.657 \\
\hline
\end{tabular}

${ }^{\varepsilon}$, two participants were counted twice for repeated diarrhea syndrome in our follow-up. *, classified into 3 groups: non-diarrhea, infection, and gut aGVHD. ${ }^{\dagger}, \mathrm{P}$ values are calculated between infection and aGVHD groups, which are 2-sided and based on Fisher's exact test. ${ }^{\text {, }}$ with reference to the clinical stage of acute GVHD (6). Grade 0: $<10 \mathrm{~mL} / \mathrm{kg}$ per day; grade I: 10-19.9 mL/kg per day; grade II: 20-30 mL/kg per day; grade III $>30 \mathrm{~mL} / \mathrm{kg}$ per day. ", C-reactive protein is defined as normal below $8 \mathrm{mg} / \mathrm{L}$. N, number; aGVHD, acute graft-versus-host disease.

( $\mathrm{P}=0.007)$ but similar to that of the normal child group. PostHSCT subjects were classified into 3 groups based on their clinical symptoms of diarrhea, and the lowest diversity was observed in the gut aGVHD group compared to that in the non-diarrhea $(\mathrm{P}=0.032)$ and intestinal infection $(\mathrm{P}=0.063)$ groups. Moreover, microbial structure and components among groups shared similarity to those in the other groups from beta diversity analysis (Figure 6B). In conclusion, we found species diversity changes in the pre- and post-HSCT groups and diarrhea groups, which hopefully suggested microbiome involvement in both intestinal infection and gut aGVHD and a therapeutic target for diarrhea after HSCT.

\section{Some bacterial LDA scores significantly differed among groups}

To determine whether the presence or absence of specific bacterial taxa correlated with patient survival or diarrhea classification, we compared microbiota relative-abundances between groups according to linear discriminant analysis.
As shown in Figure 7, 6 kinds of bacteria were found to significantly differ among the survival groups and five among the diarrhea groups. In terms of survival, a richer relative abundance of Moraxellaceae in the intestinal flora after HSCT was conducive to survival, while that of Enterococcaceae and Alphaproteobacteria was not. Similarly, a rich relative abundance of Proteobacteria, Gammaproteobacteria and Odoribacteraceae in the intestinal flora before HSCT contributed to patient death thereafter (Figure 7A). In regard to diarrhea classification, Peptostreptococcaceae, Bifidobacteriales and Bifidobacteriaceae were richer in relative abundance in the intestinal infection group after HSCT. The GVHD group had increased relative abundances of Pasteurellales and Pasteurellaceae (Figure 7B).

\section{Pasteurellaceae and Bifidobacteriaceae had high correlation coefficients with clinical characteristics}

To determine relationships between the abovementioned 


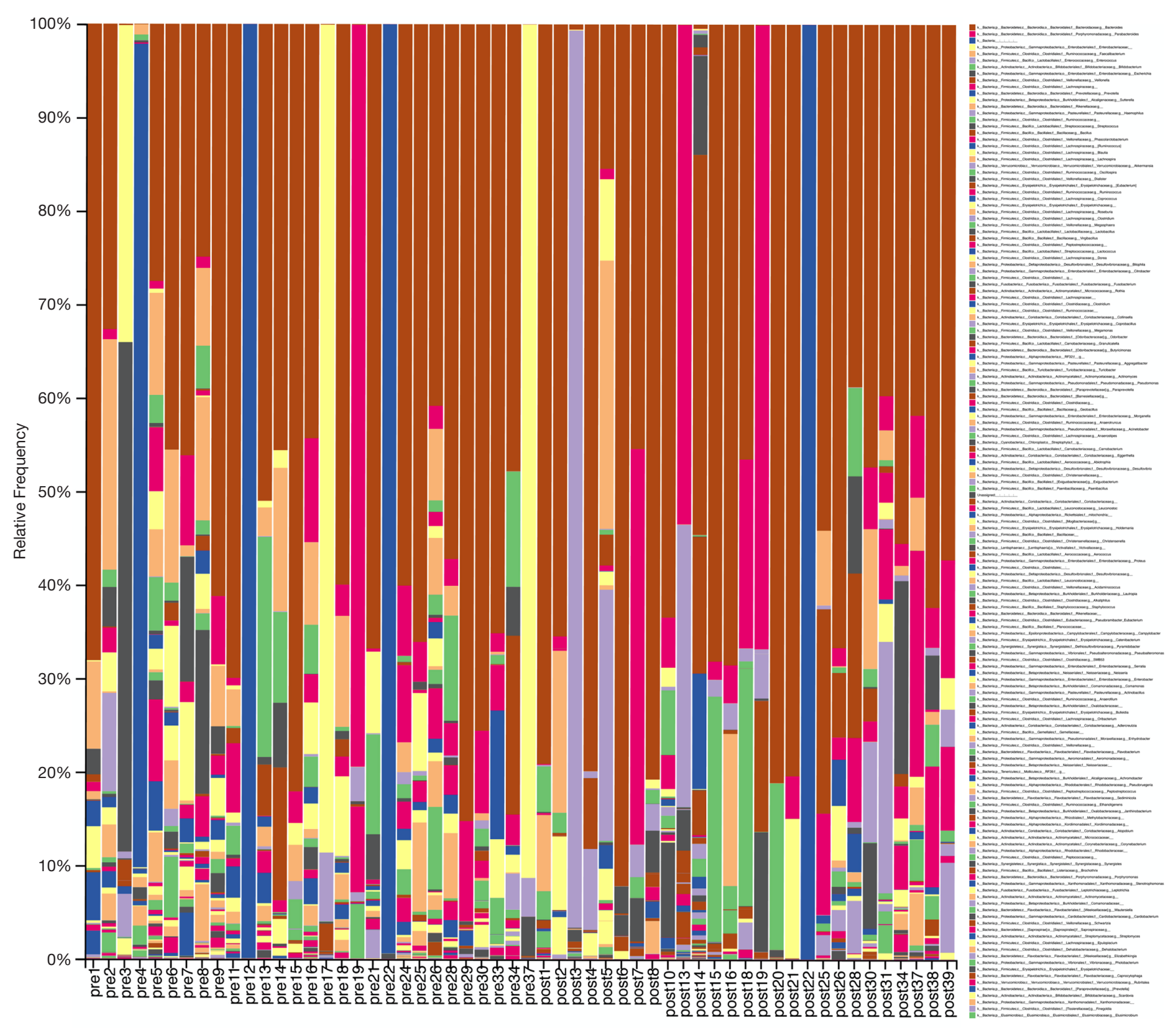

Sample

Figure 5 Taxonomy-bar-plots of all samples.

bacteria from post-HSCT samples and clinical characteristics, we then calculated their correlation coefficients (Figure 7C). Pasteurellaceae was strongly related to diarrhea classification, severity, and volume, which might refer to a more severe clinical manifestation and worse outcome for GVHD patients, demonstrating Spearman correlation coefficients of 0.648 and 0.515 and Pearson correlation coefficient of 0.599 , respectively. Bifidobacteriaceae appeared to have a close relationship with HSCT donors' and recipients' HLA matching and patients' underlying diseases, with Spearman correlation coefficients of 0.501 and 0.557 , respectively. There were also correlations of bacteria to sex, age, and diarrhea periods. In other words, some particular species of bacteria might play a role in diarrhea after HSCT, and further study on bacterial immunology might present a different view of the gut aGVHD mechanism. 

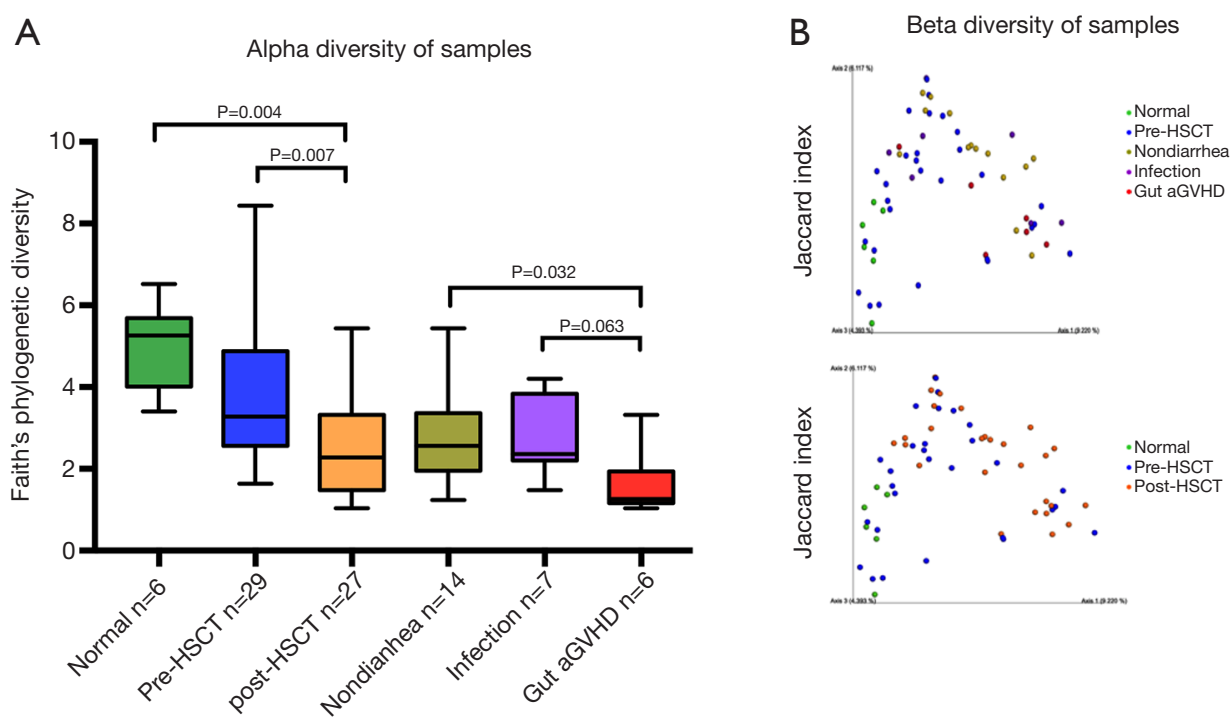

Figure 6 Alpha diversity box plot and beta diversity 2D dot plot. N, number; P, P value; HSCT, hematopoietic stem cell transplantation; aGVHD, acute graft-versus-host disease.

\section{A higher alpha diversity of post-HSCT feces led to a better survival rate}

Finally, OS was estimated from 3 diversity groups, as measured by the inverse Simpson index: high diversity, $>4$; intermediate diversity, 2 to 4 ; and low diversity, $<2$. Of the 27 participants whose samples were collected after HSCT, a total of 4 patients died. The length of follow-up was 34 to 767 days for OS. No significant differences among the 3 diversity groups $(\mathrm{P}=0.724)$ were found. However, a trend of a better survival rate was observed: the estimated OS at 3 years was $90.9 \%$ for the high diversity group and $80 \%$ for the intermediate and low diversity groups (Figure 8).

\section{Discussion}

Our study results share similarity to those of former studies in adults showing that specific dominant bacteria could interfere with the progression of diarrhea. From a clinical perspective, a 16S RNA sequencing panel of fecal bacteria may potentially help the diagnosis, treatment, and prognosis of diarrhea after HSCT. As far as our study is concerned, Bifidobacteriaceae, Pasteurellaceae and Moraxellaceae are convincing biomarkers for differentiation diagnosis and prognosis.

In this study, the abundance changes in Bifidobacteriaceae after HSCT, which was strongly dominant in infected stool samples, were associated with underlying diseases and HLA matching donor stem cell sources, which could be explained as the secondary infection of a positive immunosuppression therapy for the recipients of HLA-mismatched HSCT. Different stem cell sources have a great impact on the decision of transplantation therapy, from the intensity of the regimen to immunosuppressive agent choices after engraftment. Bifidobacteriaceae has been reported as a symbol of intestinal infection in many previous articles and reports (10). From our perspective, a rising abundance of Bifidobacteriaceae in the infectious diarrhea group verified that a positive response to anti-infectious treatment clinically supported an infection diagnosis for pathogennegative cases. However, whether Bifidobacteriaceae can be used as a diagnostic biomarker for patients suspected of infection with no evidence remains to be verified.

In addition to the diagnosis of GVHD, we found an increasing Pasteurellaceae abundance in the GVHD group that strongly worsened the severity of diarrhea, whose mechanism might be related to bacterial metabolites. Because of its distinguishing abundance from groups, Pasteurellaceae might play a role in differentiation of gut aGVHD from infectious diarrhea.

Furthermore, we did find a family of bacteria, Moraxellaceae, that might benefit survival after HSCT. Moraxellaceae was also correlated with HLA matching, according to the results, which influenced the decline in immune function by a fully or partially myeloablative immunosuppressive regimens. Along 


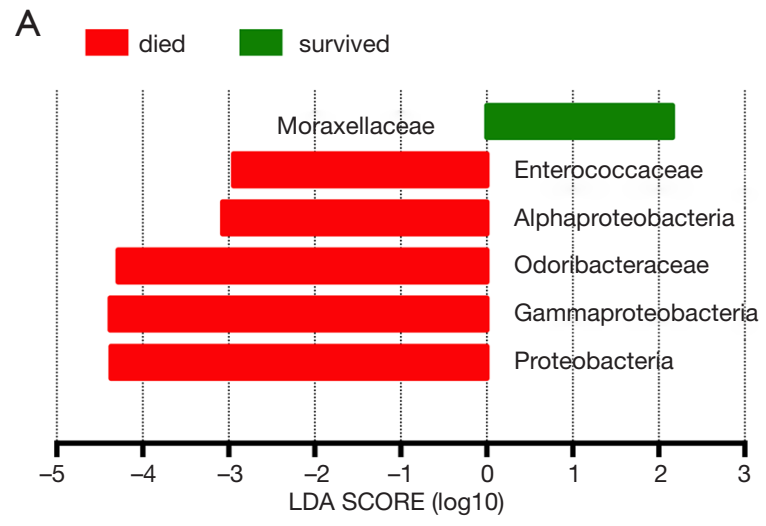

B

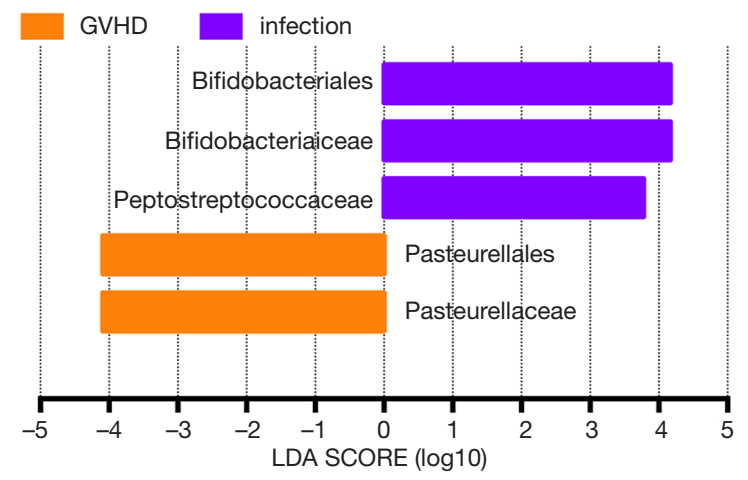

C

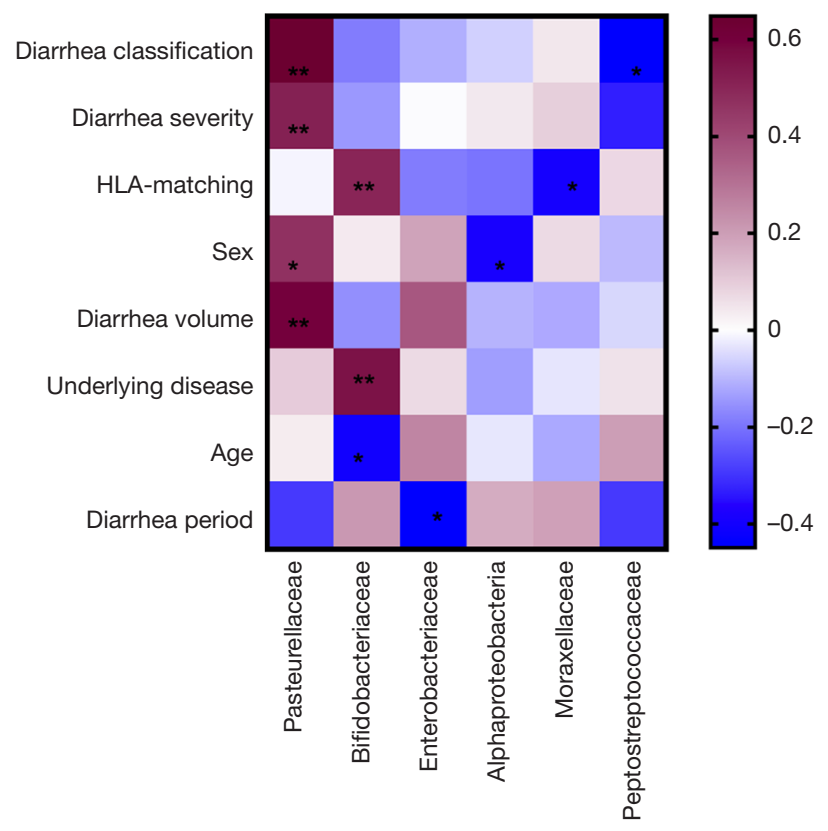

Figure 7 Histogram of LDA score between groups and heatmap of the coefficient of correlation between the bacterial flora and diarrhea manifestations. LDA, linear discriminant analysis; GVHD, graft-versus-host disease; HLA, human leukocyte antigen.

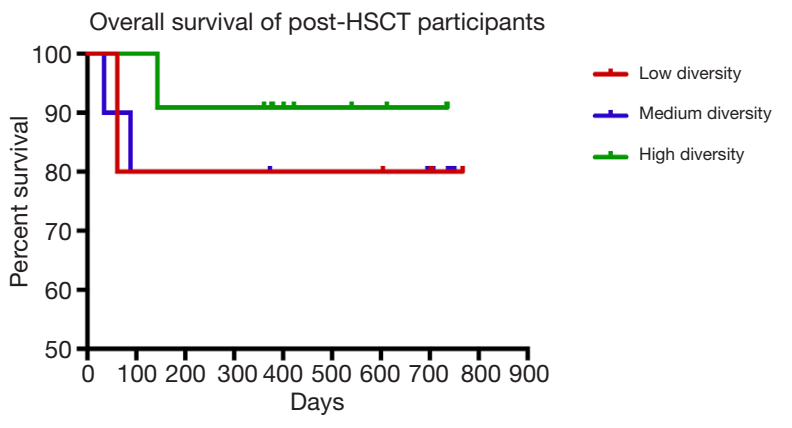

Figure 8 Kaplan-Meier survival curves of post-HSCT participants with different microbial diversities*. *, one surviving child had 2 posttransplant samples, and the diversity data at the time of +1 month after transplantation were taken as a reference. HSCT, hematopoietic stem cell transplantation. to the immunosuppression, the engraftment rate after transplantation (11,12), GVHD occurrence (13) and cytomegalovirus (CMV) or other viral infections $(14,15)$ will be affected by different types of HLA-matching donors. The most direct influence is the killing of intestinal mucosal tissue by donor $\mathrm{T}$ cells (16), which causes intestinal GVHD and worsens infection. In the case of GVHD, the intestinal mucosal barrier is destroyed due to apoptosis of intestinal epithelial cells and ectopic growth of intestinal bacteria that aggravates the inflammatory response (17), of which one classical theory is the apoptosis of Paneth cells inhibiting the NOTCH signaling pathway and downregulating the expression of EGF, IL-22 and other cytokines that inhibit GVHD $(18,19)$. We believe that Moraxellaceae, frequently 
found in food products with weak proteolytic activities and few offensive byproducts (20), might play an inhibiting role in inflammatory responses after HSCT. Thus, we believe that there is additional convincing evidence that the intestinal flora is involved in the production and functional effects of immune cells.

Diarrhea after HSCT is such a complicated problem that a single bacterial panel could suggest only the potential causes. We still need to find additional evidence for diagnosis. As shown in our results, the decrease in intestinal flora diversity can largely indicate the occurrence of gut aGVHD. The alpha diversity after transplantation was significantly lower than that before transplantation, reflecting the sensitivity of the intestinal flora to the conditioning regimen to some extent (21). Among them, the alpha diversity of samples from children with GVHD was significantly lower than that of other posttransplant samples, further suggesting that the intestinal flora was further unbalanced due to barrier destruction during GVHD and fell into a vicious circle of "decreased microbial diversity-GVHD aggravation”. In recent years, a number of studies have proposed the predictive effect of microbial diversity on the OS rate through the analysis of fecal samples at specific time points $(2,3)$. In addition, it has been reported that in a mouse model, a reduction in bacterial diversity causes a delay in lymphoid and neutrophil hematopoietic reconstitution (22), which increases the risks of infection, engraftment failure and death. Although an effect of diversity change on survival rate was not seen in this study, the superiority of the high diversity group in survival rate was still obvious.

When it comes to the microbiological treatment, fecal microbiome transplantation (FMT) technology has achieved satisfactory results in areas such as refractory C. difficile infection (rCDI) (23) and inflammatory bowel disease (IBD) (24). We also saw multiple FMT case reports of immunodeficiency patients (25) and CDI infections in children after HSCT transplantation (26,27). In this study, the alpha diversity of children in the GVHD group was significantly reduced, providing a theoretical basis for FMT in gut aGVHD. Kakihana, DeFilipp $(28,29)$ and others also reported GVHD-related FMT treatment in adults that some patients with severe GVHD symptoms were even completely relieved. An unpublished FMT case research from our center also suggested that the bacterial diversity of gut aGVHD patients after FMT was improved and the distribution of flora was changed.
Intestinal microbiome research in the field of HSCT has been applied for decades. As research progresses, we have a better understanding of specific pathogen infections $(30,31)$, antibiotic-associated microbiota imbalance (31) and participation in the mechanism of HSCT. We suggest a new diagnostic process combining a 16S RNA fecal bacteria panel with microbiome diversity analysis. Since this is a single-center study, which limits the sample size, whether this new method of differentiation diagnosis is effective remains to be discussed. Additionally, due to the large scale of underlying diseases contained in this study, the prognosis after HSCT and sensitivity of intestinal symptoms might inevitably influence the outcome. Further studies on the immune effects of the microbiome when GVHD occurs, especially related to the pathogenesis of GVHD, may introduce a brand-new regimen of flora reconstruction due to GVHD arrangement and take advantage of improved outcomes for pediatric HSCT patients.

\section{Conclusions}

The gut microbiota diversity was lowest when gut aGVHD occurred, which was consistent with the clinically higher mortality rate and greater treatment difficulty. Specific species may affect the severity of diarrhea, and stem cell sources may also affect the composition of the flora. With the development of pediatric HSCT, complications after transplantation have been proven to be an individual factor in the success rate of engraftment. Clinicians are working hard on all complications for a better survival rate. Intestinal complications are familiar but still difficult in some severe cases. Owing to progress in microbiome studies, we can take a different perspective on gut aGVHD and intestinal infection.

\section{Acknowledgments}

The authors would like to thank Haoyan Chen, a researcher of Shanghai Cancer Institute.

Funding: This work was supported by the Clinical Science and Technology Innovation Project of the Development Center of Shanghai Shenkang Hospital (SHDC12019X35), the Research Project of the Collaborative Innovation Centre of Translational Medicine in Shanghai Jiao Tong University (TM201820), National Natural Science Foundation of China (81900812 to NS) and the Interdisciplinary Program of Shanghai Jiao Tong University (YG2017QN33 to NS). 


\section{Footnote}

Reporting Checklist: The authors have completed the STROBE reporting checklist. Available at http://dx.doi. org/10.21037/tp-20-208

Data Sharing Statement: Available at http://dx.doi. org/10.21037/tp-20-208

Conflicts of Interest: All authors have completed the ICMJE uniform disclosure form (available at http://dx.doi. org/10.21037/tp-20-208). The authors have no conflicts of interest to declare.

Ethical Statement: The authors are accountable for all aspects of the work in ensuring that questions related to the accuracy or integrity of any part of the work are appropriately investigated and resolved. The study was conducted in accordance with the Declaration of Helsinki (as revised in 2013). The studies involving human participants were reviewed and approved by the Human Ethics Committee of Shanghai Children's Medical Center (No.: SCMCIRB-K2016053-2). Written informed consent to participate in this study was provided by the participants' legal guardian/next of kin.

Open Access Statement: This is an Open Access article distributed in accordance with the Creative Commons Attribution-NonCommercial-NoDerivs 4.0 International License (CC BY-NC-ND 4.0), which permits the noncommercial replication and distribution of the article with the strict proviso that no changes or edits are made and the original work is properly cited (including links to both the formal publication through the relevant DOI and the license). See: https://creativecommons.org/licenses/by-nc-nd/4.0/.

\section{References}

1. Naymagon S, Naymagon L, Wong SY, et al. Acute graftversus-host disease of the gut: Considerations for the gastroenterologist. Nat Rev Gastroenterol Hepatol 2017;14:711-26.

2. Golob JL, Pergam SA, Srinivasan S, et al. Stool microbiota at neutrophil recovery is predictive for severe acute graft vs host disease after hematopoietic cell transplantation. Clin Infect Dis 2017;65:1984-91.

3. Taur Y, Jenq RR, Perales MA, et al. The effects of intestinal tract bacterial diversity on mortality following allogeneic hematopoietic stem cell transplantation. Blood 2014;124:1174-82.

4. Vossen JM, Guiot HF, Lankester AC, et al. Complete suppression of the gut microbiome prevents acute graftversus-host disease following allogeneic bone marrow transplantation. PLoS One 2014;9:e105706.

5. Dominianni C, Sinha R, Goedert JJ, et al. Sex, body mass index, and dietary fiber intake influence the human gut microbiome. PLoS One 2015;10:e0124599.

6. Rowlings PA, Przepiorka D, Klein JP, et al. IBMTR Severity Index for grading acute graft-versus-host disease: Retrospective comparison with Glucksberg grade. Br J Haematol 1997;97:855-64.

7. Ruutu T, Gratwohl A, de Witte T, et al. Prophylaxis and treatment of GVHD: EBMT-ELN working group recommendations for a standardized practice. Bone Marrow Transplant 2014;49:168-73.

8. Working Group on Obesity in China. Body mass index reference norm for screening overweight and obesity in Chinese children and adolescents. Zhonghua Liu Xing Bing Xue Za Zhi 2004;25:97-102.

9. Schloss PD, Westcott SL, Ryabin T, et al. Introducing mothur: open-source, platform-independent, communitysupported software for describing and comparing microbial communities. Appl Environ Microbiol 2009;75:7537-41.

10. Lynch SV, Goldfarb KC, Wild YK, et al. Cystic fibrosis transmembrane conductance regulator knockout mice exhibit aberrant gastrointestinal microbiota. Gut Microbes 2013;4:41-7.

11. Klein OR, Buddenbaum J, Tucker N, et al. Nonmyeloablative haploidentical bone marrow transplantation with post-transplantation cyclophosphamide for pediatric and young adult patients with high-risk hematologic malignancies. Biol Blood Marrow Transplant 2017;23:325-32.

12. Mahadeo KM, Weinberg KI, Abdel-Azim H, et al. A reduced-toxicity regimen is associated with durable engraftment and clinical cure of nonmalignant genetic diseases among children undergoing blood and marrow transplantation with an HLA-matched related donor. Biol Blood Marrow Transplant 2015;21:440-4.

13. McCurdy SR, Kanakry CG, Tsai HL, et al. Grade II acute graft-versus-host disease and higher nucleated cell graft dose improve progression-free survival after HLA-haploidentical transplant with post-transplant cyclophosphamide. Biol Blood Marrow Transplant 2018;24:343-52.

14. Shams El-Din AA, El-Desoukey NA, Amin Tawadrous 
DG, et al. The potential association of CMV-specific CD8+ T lymphocyte reconstitution with the risk of CMV reactivation and persistency in post allogeneic stem cell transplant patients. Hematology 2018;23:463-9.

15. Takenaka K, Gondo H, Tanimoto K, et al. Increased incidence of cytomegalovirus (CMV) infection and $\mathrm{CMV}$-associated disease after allogeneic bone marrow transplantation from unrelated donors. The Fukuoka bone marrow transplantation group. Bone Marrow Transplant 1997;19:241-8.

16. Zeiser R, Nguyen VH, Beilhack A, et al. Inhibition of $\mathrm{CD} 4+\mathrm{CD} 25+$ regulatory $\mathrm{T}$-cell function by calcineurin-dependent interleukin-2 production. Blood 2006;108:390-9.

17. Eriguchi Y, Takashima S, Oka H, et al. Graft-versushost disease disrupts intestinal microbial ecology by inhibiting Paneth cell production of $\alpha$-defensins. Blood 2012;120:223-31.

18. Levine JE, Huber E, Hammer ST, et al. Low Paneth cell numbers at onset of gastrointestinal graft-versushost disease identify patients at high risk for nonrelapse mortality. Blood 2013;122:1505-9.

19. Sato T, van Es JH, Snippert HJ, et al. Paneth cells constitute the niche for Lgr5 stem cells in intestinal crypts. Nature 2011;469:415-8.

20. Yang X. Moraxellaceae. In: Batt CA, Tortorello ML, editors. Encyclopedia of food microbiology. 2nd ed. Cambridge, US: Academic Press, 2014:826-33.

21. Montassier E, Batard E, Massart S, et al. 16S rRNA gene pyrosequencing reveals shift in patient faecal microbiota during high-dose chemotherapy as conditioning regimen for bone marrow transplantation. Microb Ecol 2014;67:690-9.

22. Staffas A, Burgos da Silva M, Slingerland AE, et al. Nutritional support from the intestinal microbiota improves hematopoietic reconstitution after bone marrow transplantation in mice. Cell Host Microbe 2018;23:447-57.e4.

Cite this article as: Song A, Shen N, Gan C, Luo C, Luo C, Wang J, Cao Q, Chen J. Exploration of the relationship between intestinal flora changes and gut acute graft-versus-host disease after hematopoietic stem cell transplantation. Transl Pediatr 2021;10(2):283-295. doi: 10.21037/tp-20-208
23. Kassam Z, Lee CH, Yuan Y, et al. Fecal microbiota transplantation for Clostridium difficile infection: systematic review and meta-analysis. Am J Gastroenterol 2013;108:500-8.

24. M N Quraishi, T Critchlow, N Bhala, et al. Faecal transplantation for IBD management-pitfalls and promises, Br Med Bull 2017;124:181-90.

25. Kelly CR, Ihunnah C, Fischer M, et al. Fecal microbiota transplant for treatment of Clostridium difficile infection in immunocompromised patients. Am J Gastroenterol 2014;109:1065-71.

26. Flannigan KL, Rajbar T, Moffat A, et al. Changes in Composition of the Gut Bacterial Microbiome after Fecal Microbiota Transplantation for Recurrent Clostridium difficile Infection in a Pediatric Heart Transplant Patient. Front Cardiovasc Med 2017;4:17.

27. Hira Bluestone, Matthew P Kronman, David L Suskind; Fecal Microbiota Transplantation for Recurrent Clostridium difficile Infections in Pediatric Hematopoietic Stem Cell Transplant Recipients, J Pediatric Infect Dis Soc 2018;7:e6-8.

28. Kakihana K, Fujioka Y, Suda W, et al. Fecal microbiota transplantation for patients with steroid-resistant acute graft-versus-host disease of the gut. Blood 2016;128:2083-8.

29. DeFilipp Z, Peled JU, Li S, et al. Third-party fecal microbiota transplantation following allo-HCT reconstitutes microbiome diversity. Blood Adv 2018;2:745-53.

30. Willems L, Porcher R, Lafaurie M, et al. Clostridium difficile infection after allogeneic hematopoietic stem cell transplantation: incidence, risk factors, and outcome. Biol Blood Marrow Transplant 2012;18:1295-301.

31. Shono Y, Docampo MD, Peled JU, et al. Increased GVHD-related mortality with broad-spectrum antibiotic use after allogeneic hematopoietic stem cell transplantation in human patients and mice. Sci Transl Med 2016;8:339ra71. 